\title{
Quadrature Oscillators Using Operational Amplifiers
}

\author{
Jiun-Wei Horng \\ Department of Electronic Engineering, Chung Yuan Christian University, Chung-Li 32023, Taiwan \\ Correspondence should be addressed to Jiun-Wei Horng, jwhorng@cycu.edu.tw
}

Received 19 May 2011; Accepted 2 July 2011

Academic Editor: Ahmed M. Soliman

Copyright (C) 2011 Jiun-Wei Horng. This is an open access article distributed under the Creative Commons Attribution License, which permits unrestricted use, distribution, and reproduction in any medium, provided the original work is properly cited.

Two new quadrature oscillator circuits using operational amplifiers are presented. Outputs of two sinusoidal signals with $90^{\circ}$ phase difference are available in each circuit configuration. Both proposed quadrature oscillators are based on third-order characteristic equations. The oscillation conditions and oscillation frequencies of the proposed quadrature oscillators are orthogonally controllable. The circuits are implemented using the widely available operational amplifiers which results in low output impedance and high current drive capability. Experimental results are included.

\section{Introduction}

Quadrature oscillator is used because the circuit provides two sinusoids with $90^{\circ}$ phase difference, as, for example, in telecommunications for quadrature mixers and single-sideband generators or for measurement purposes in vector generators or selective voltmeters. Therefore, quadrature oscillators constitute an important unit in many communication and instrumentation systems [1-7].

Recently, several multiphase oscillators based on operational amplifiers were proposed [6-11]. Two-integrator loop technique was developed to realize quadrature oscillators using operational amplifiers [6]. In 1993 [7], Holzel proposed a new method for realizing quadrature oscillator, which consists of two all-pass filters and one inverter using operational amplifiers. Several multiphase oscillators using operational amplifiers were proposed in [8-11]. However, the quadrature output voltages cannot be obtained from [810]. The multiphase sinusoidal oscillator in [11] was constructed by cascading several first-order all-pass networks and unity-gain inverting networks. However, the block diagram of the quadrature oscillators in [11] was the same with [7].

In this paper, two new quadrature oscillator circuits using operational amplifiers are proposed. Outputs of two sinusoidal signals with $90^{\circ}$ phase difference are available in each proposed circuit configuration. Both proposed quadrature oscillators are based on third-order characteristic equations.
The oscillation conditions and oscillation frequencies of the proposed quadrature oscillators are orthogonally controllable. The circuits are implemented using the widely available operational amplifiers which results in low output impedance, high current drive capability (enabling the systems to drive a variety of loads), simplicity, and low cost.

\section{Circuit Description}

Figure 1 shows the first proposed quadrature oscillator circuit. The characteristic equation of the circuit can be expressed as

$$
\begin{aligned}
& s^{3} C_{1} C_{2} C_{3} R_{1} R_{2} R_{3} R_{4} R_{5}+s^{2} C_{3} R_{3} R_{4} R_{5}\left(C_{1} R_{1}+C_{2} R_{2}\right) \\
& +s C_{3} R_{3} R_{4} R_{5}+R_{1} R_{2}=0 .
\end{aligned}
$$

At $s=j \omega$, by equating the real and imaginary parts with zero, the oscillation condition and oscillation frequency can be obtained as

$$
\begin{aligned}
R_{3} R_{4} R_{5} & =\frac{C_{1} C_{2} R_{1}{ }^{2} R_{2}^{2}}{C_{3}\left(C_{1} R_{1}+C_{2} R_{2}\right)}, \\
\omega_{o} & =\frac{1}{\sqrt{C_{1} C_{2} R_{1} R_{2}}} .
\end{aligned}
$$

From (2) and (3), the oscillation condition and oscillation frequency can be orthogonally controllable. 
From Figure 1, the voltage transfer function from $V_{o 2}$ to $V_{o 1}$ is

$$
\frac{V_{o 2}}{V_{o 1}}=-\frac{1}{s C_{3} R_{4}}
$$

The phase difference, $\phi$, between $V_{o 2}$ and $V_{o 1}$ is

$$
\phi=90^{\circ}
$$

ensuring the voltage $V_{o 2}$ and $V_{o 1}$ to be in quadrature. Because the output impedance of the operational amplifier is very small, the two output terminals, $V_{o 1}$ and $V_{o 2}$, can be directly connected to the next stage, respectively.

The passive sensitivities of the quadrature oscillator in Figure 1 are all low and obtained as

$$
S_{C_{1}, C_{2}, R_{1}, R_{2}}^{\omega_{o}}=-\frac{1}{2}
$$

Figure 2 shows the second proposed quadrature oscillator circuit. The characteristic equation of the circuit can be expressed as

$$
\begin{aligned}
& s^{3} C_{1} C_{2} C_{3} C_{4} C_{5} R_{1} R_{2} R_{3}+s^{2} C_{3} C_{4} C_{5} R_{3}\left(C_{1} R_{1}+C_{2} R_{2}\right) \\
& \quad+s C_{3} C_{4} C_{5} R_{3}+C_{1} C_{2}=0 .
\end{aligned}
$$

At $s=j \omega$, by equating the real and imaginary parts with zero, the oscillation condition and oscillation frequency can be obtained as

$$
\begin{gathered}
R_{3}=\frac{C_{1}{ }^{2} C_{2}{ }^{2} R_{1} R_{2}}{C_{3} C_{4} C_{5}\left(C_{1} R_{1}+C_{2} R_{2}\right)}, \\
\omega_{o}=\frac{1}{\sqrt{C_{1} C_{2} R_{1} R_{2}}} .
\end{gathered}
$$

From (8) and (9), the oscillation condition and oscillation frequency can be orthogonally controllable. $V_{o 1}$ is

From Figure 2, the voltage transfer function from $V_{o 2}$ to

$$
\frac{V_{o 2}}{V_{o 1}}=-\frac{1}{s C_{3} R_{3}} .
$$

The phase difference, $\phi$, between $V_{o 2}$ and $V_{o 1}$ is

$$
\phi=90^{\circ}
$$

ensuring the voltage $V_{o 2}$ and $V_{o 1}$ to be in quadrature. Because the output impedance of the operational amplifier is very small, the two output terminals, $V_{o 1}$ and $V_{o 2}$, can be directly connected to the next stage, respectively.

The passive sensitivities of the quadrature oscillator in Figure 2 are all low and obtained as

$$
S_{C_{1}, C_{2}, R_{1}, R_{2}}^{\omega_{o}}=-\frac{1}{2}
$$

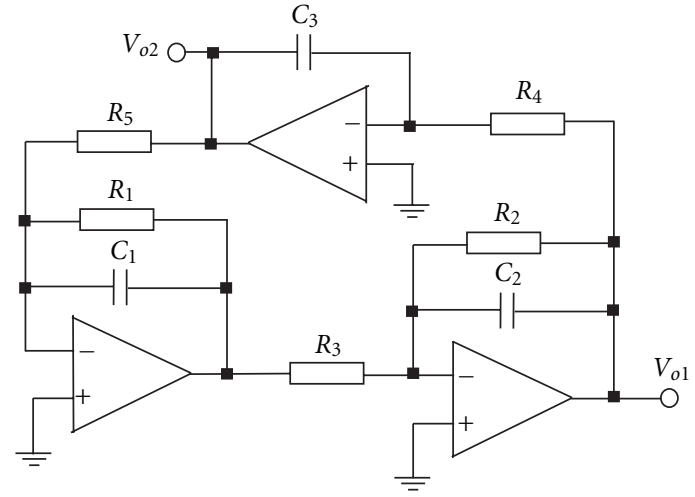

FIgURE 1: The first proposed quadrature oscillator circuit.

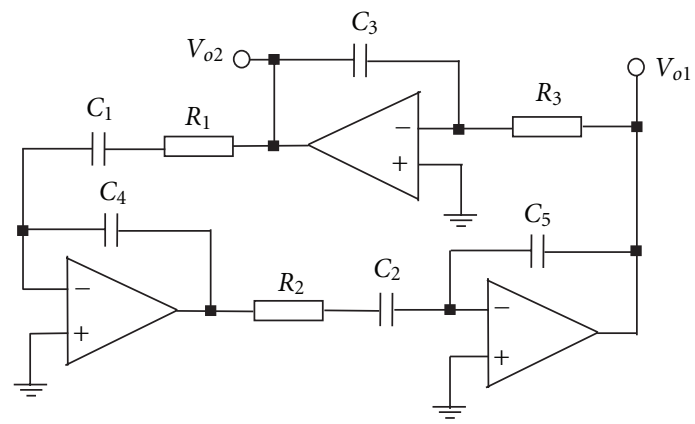

FIgURE 2: The second proposed quadrature oscillator circuit.

\section{Experimental Results}

The quadrature oscillator in Figure 1 was constructed using LF351s. Figure 3 represents the quadrature sinusoidal output waveforms of Figure 1 with $C_{1}=C_{2}=C_{3}=1 \mathrm{nF}, R_{1}=R_{2}=R_{4}$ $=R_{5}=10 \mathrm{k} \Omega, R_{3}=4.563 \mathrm{k} \Omega$, and the power supply $\pm 10 \mathrm{~V}$. Figure 4 shows the experimental results of the oscillation frequency of Figure 1 by varying the value of $R\left(R=R_{1}=\right.$ $R_{2}=R_{4}=R_{5}$ ) with $C_{1}=C_{2}=C_{3}=1 \mathrm{nF}$, and $R_{3}$ was varied with $R$ by (2) to ensure the oscillations will start.

The quadrature oscillator in Figure 2 was constructed using LF351s. Figure 5 represents the quadrature sinusoidal output waveforms of Figure 2 with $C_{1}=C_{2}=C_{3}=C_{4}=$ $C_{5}=1 \mathrm{nF}, R_{1}=R_{2}=10 \mathrm{k} \Omega, R_{3}=4.767 \mathrm{k} \Omega$, and the power supply $\pm 10 \mathrm{~V}$. Figure 6 shows the experimental results of the oscillation frequency of Figure 2 by varying the value of $R$ ( $R$ $=R_{1}=R_{2}$ ) with $C_{1}=C_{2}=C_{3}=C_{4}=C_{5}=1 \mathrm{nF}$, and $R_{3}$ was varied with $R$ by (8) to ensure the oscillations will start.

\section{Conclusions}

Two new quadrature oscillator circuits based on operational amplifiers are presented. The proposed quadrature oscillators provide the following advantages: (i) two sinusoidal output signals of $90^{\circ}$ phase difference are obtained simultaneously in each configuration; (ii) the oscillation conditions and oscillation frequencies are orthogonally controllable; (iii) the output terminals have the advantages of low output 


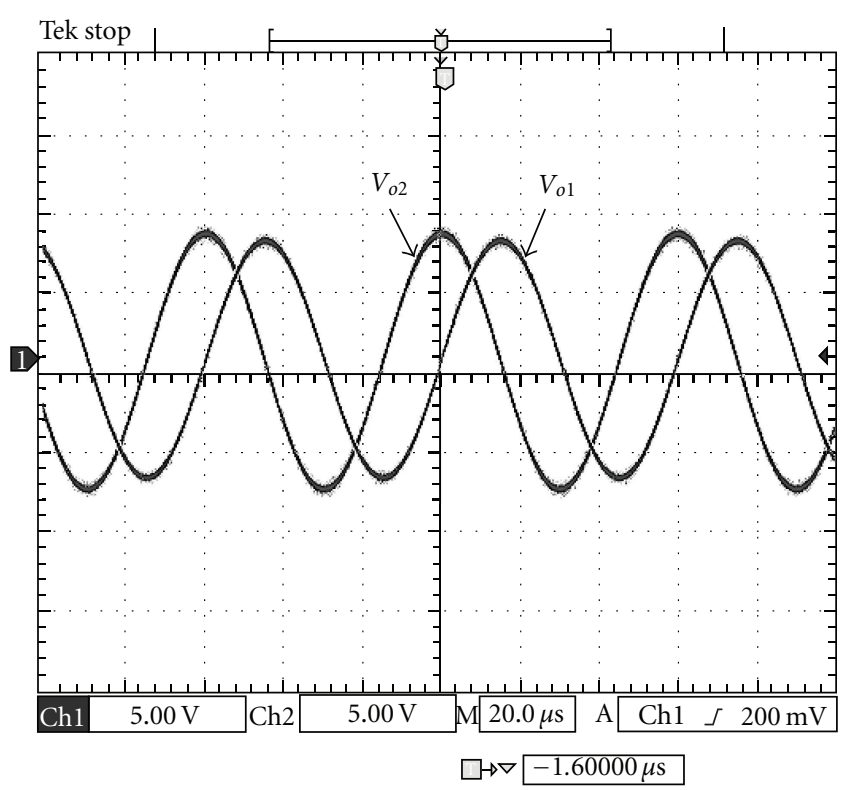

Figure 3: The experimental quadrature output waveforms of Figure 1 .

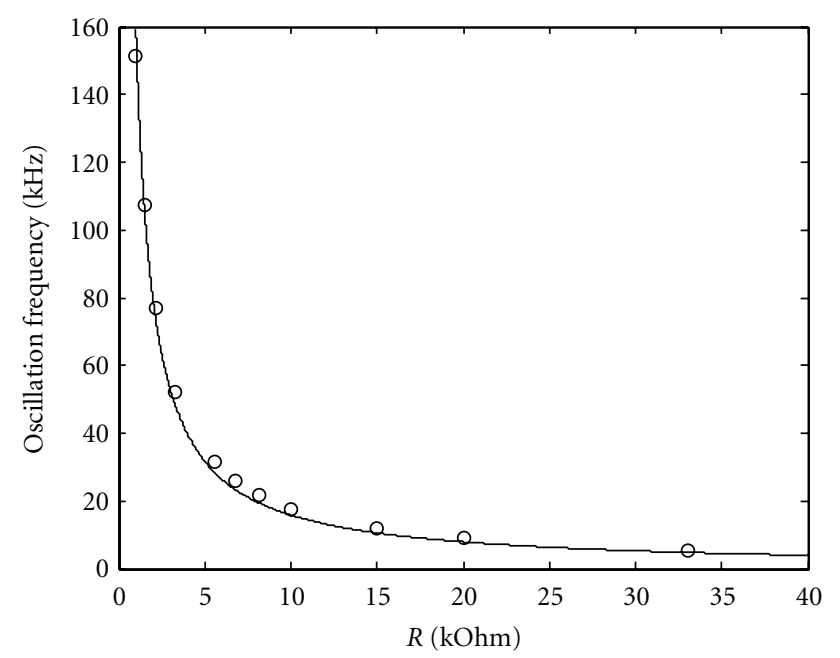

FIgURE 4: Experimental results of the oscillation frequency of Figure 1, which is obtained by varying the value of R; o o o, experimental results; - , ideal curve.

impedances and high current drive capability; (iv) simplicity and low cost; (v) the passive sensitivities are low.

\section{References}

[1] M. T. Ahmed, I. A. Khan, and N. Minhaj, "On transconductance-C quadrature oscillators," International Journal of Electronics, vol. 83, no. 2, pp. 201-207, 1997.

[2] J. W. Horng, "Current differencing buffered amplifiers based single resistance controlled quadrature oscillator employing grounded capacitors," IEICE Transactions on Fundamentals of Electronics, Communications and Computer Sciences, vol. E85A, no. 6, pp. 1416-1419, 2002.

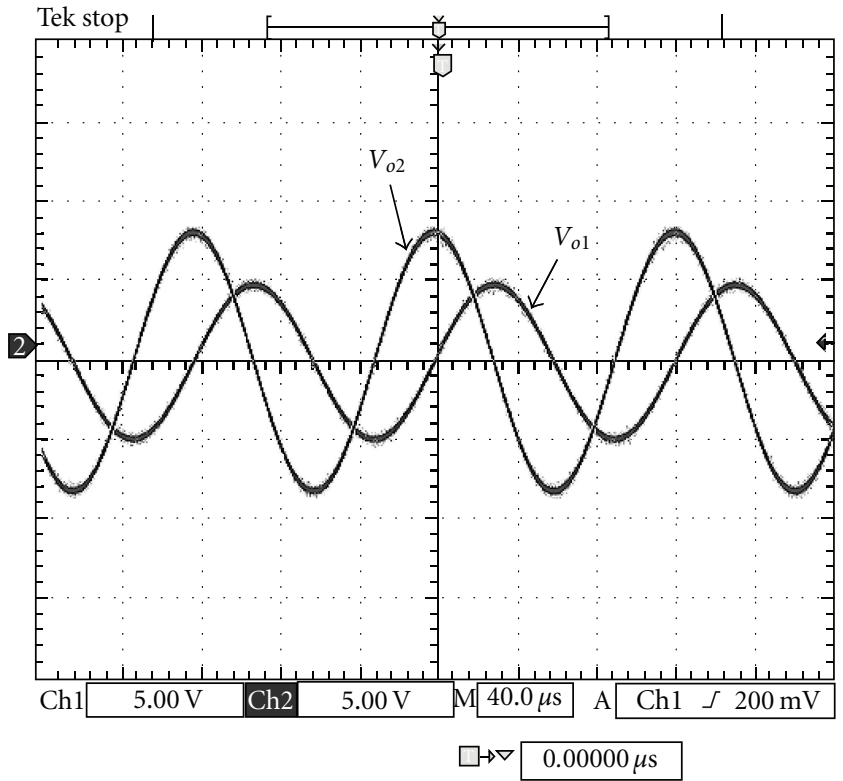

FIGURE 5: The experimental quadrature output waveforms of Figure 2.

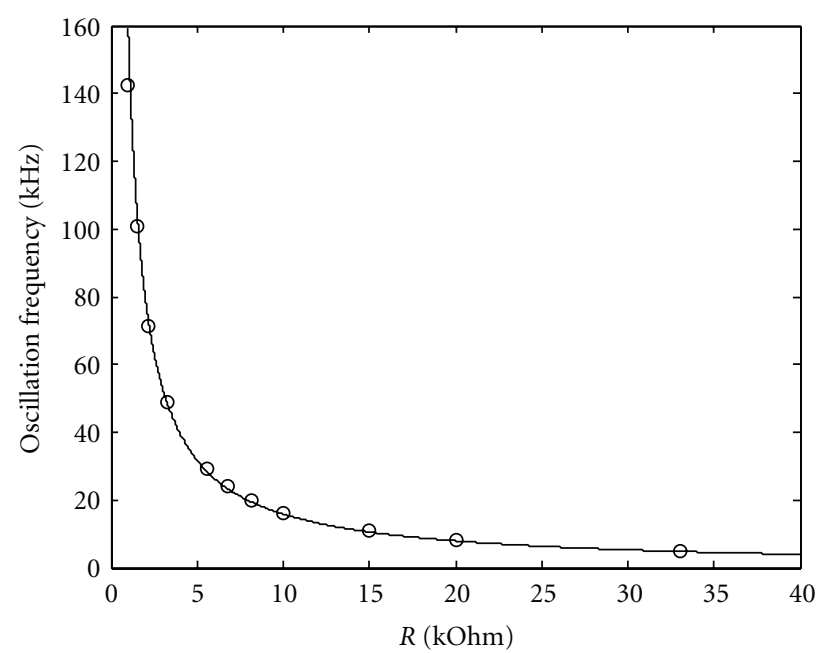

FIGURE 6: Experimental results of the oscillation frequency of Figure 2, which is obtained by varying the value of R; o o o, experimental results; - , ideal curve.

[3] M. Kumngern and K. Dejhan, "DDCC-based quadrature oscillator with grounded capacitors and resistors," Active and Passive Electronic Components, vol. 2009, Article ID 987304, 2009.

[4] J. W. Horng, H. Lee, and J. Y. Wu, "Electronically tunable third-order quadrature oscillator using CDTAs," Radioengineering, vol. 19, no. 2, pp. 326-330, 2010.

[5] W. Tangsrirat and W. Tanjaroen, "Current-mode sinusoidal quadrature oscillator with independent control of oscillation frequency and condition using CDTAs," Indian Journal of Pure and Applied Physics, vol. 48, no. 5, pp. 363-366, 2010.

[6] A. S. Sedra and K. C. Smith, Microelectronic Circuits, Oxford University Press, New York, NY, USA, 4th edition, 1998. 
[7] R. Holzel, "Simple wide-band sine wave quadrature oscillator," IEEE Transactions on Instrumentation and Measurement, vol. 42, no. 3, pp. 758-760, 1993.

[8] M. T. Abuelma'atti and W. A. Almansoury, "Active-R multiphase oscillators," IEE Proceedings Part G, vol. 134, no. 6, pp. 292-294, 1987.

[9] S. J. G. Gift, "Multiphase sinusoidal oscillator system using operational amplifiers," International Journal of Electronics, vol. 83, no. 1, pp. 61-67, 1997.

[10] S. J. G. Gift, "Multiphase sinusoidal oscillator using invertingmode operational amplifiers," IEEE Transactions on Instrumentation and Measurement, vol. 47, no. 4, pp. 986-991, 1998.

[11] S. J. G. Gift, "Application of all-pass filters in the design of multiphase sinusoidal systems," Microelectronics Journal, vol. 31, no. 1, pp. 9-13, 2000. 

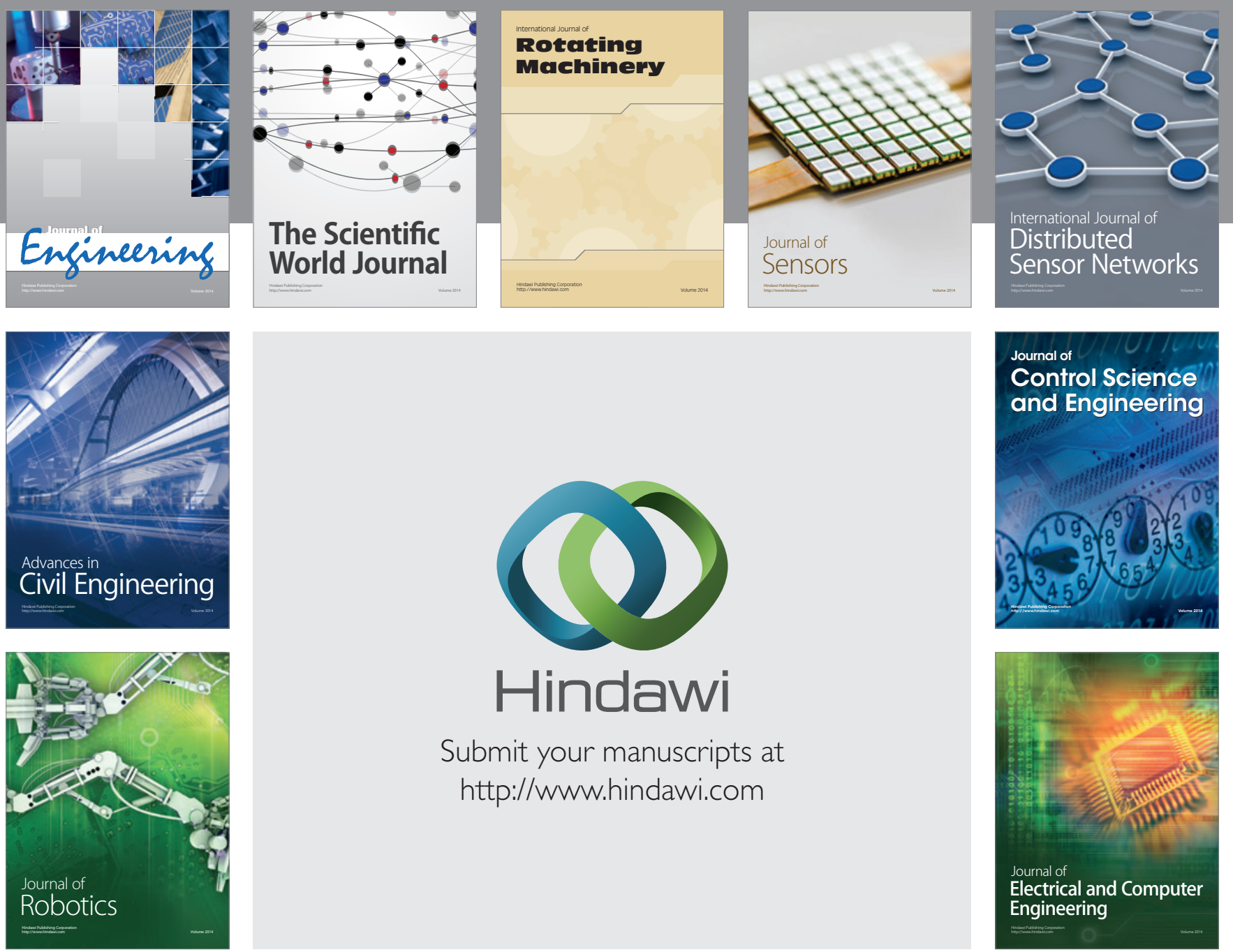

Submit your manuscripts at

http://www.hindawi.com
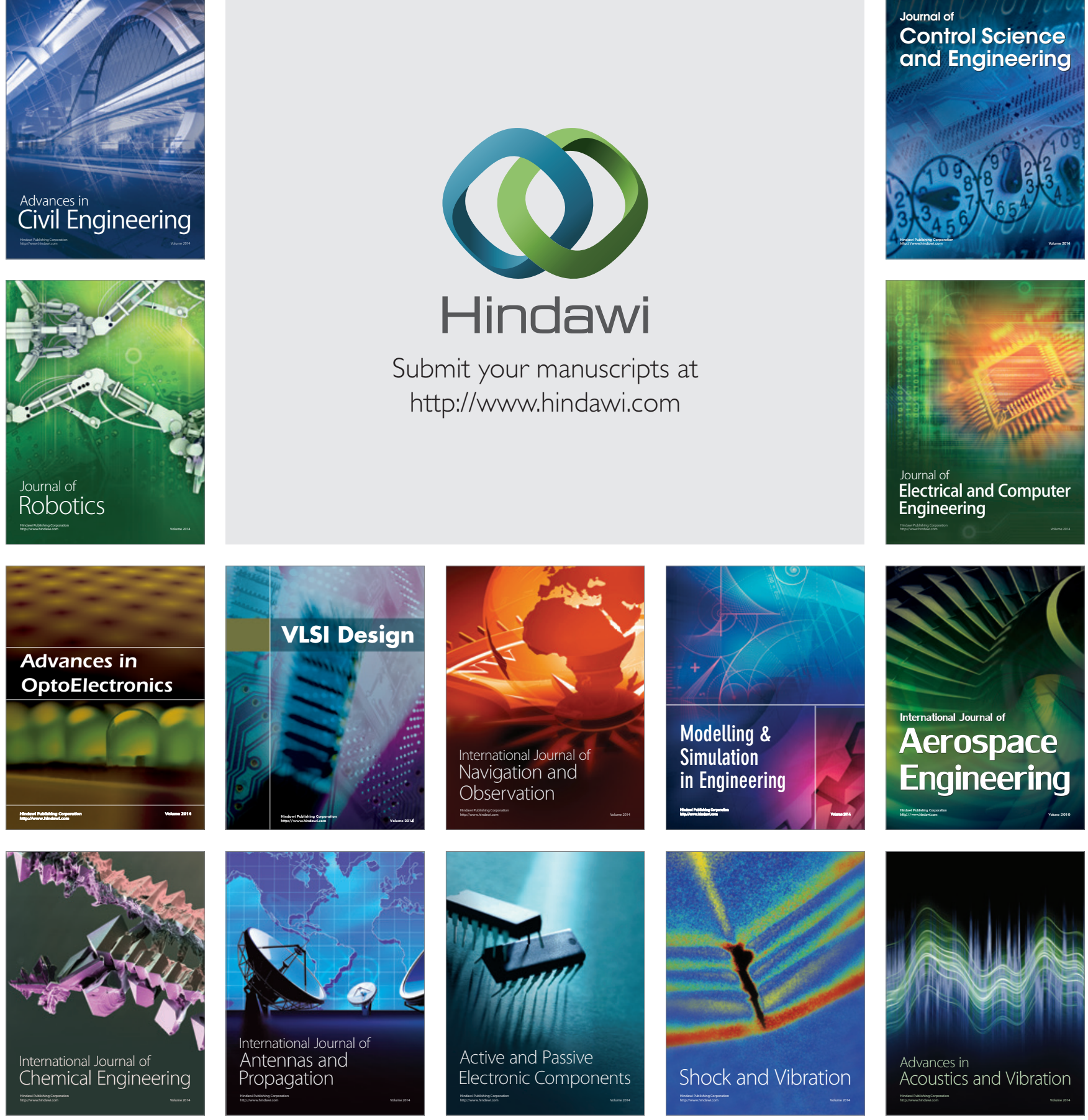